\title{
Perfil de competencias del director de recursos humanos en empresas guayaquilleñas
}

\author{
José García Arroyo / Maria lleana Cevallos Ycaza
}

\section{RESUMEN}

Este estudio exploratorio presenta el perfil de competencias del director de recursos humanos de empresas guayaquileñas. Mediante una encuesta se pidió a los directores de recursos humanos participantes que seleccionaran, de una lista, las competencias que consideraban relevantes para su desempeño, y las ordenaran según su importancia de 1 a 10, siendo 1 la más importante. La muestra estuvo conformada por 122 directores de recursos humanos de empresas pequeñas, medianas y grandes, de los tres sectores de actividad. Se realizó un análisis de frecuencias para obtener la relevancia de las competencias y se consideraron variables como el género, experiencia laboral, nivel de estudios y tipo de empresa. Los resultados muestran que los directores identificaron cinco competencias como "claves" para su desempeño. Estas son en orden de importancia: liderazgo, desarrollo del personal, habilidades de comunicación, planificación/organización y negociación. Para cada competencia se señalan comportamientos asociados seleccionados usando la misma metodología.

\section{PALABRAS CLAVE}

Perfil de competencias, ocupación, director de recursos bumanos, comportamientos, empresas guayaquileñas.

\section{ABSTRACT}

This quantitative study explores the competency profile of Human Resources Directors from Guayaquil (Ecuador). Using a survey participants were asked to rate a list of competencies in order of importance from 1 to 10; 1 being most important. The sample consisted of 122 human resources managers, among small, medium and large companies. The data was analyzed for statistical frequency and variables such as gender, work experience, and education were considered. The results show that the directors identified five competencies as being critical for effectiveness in their performance. In order of importance, the competencies are: leadership; staff development; communication skills; planning/organization; and negotiation. Behaviors associated with each competency were also identified using the same methodology.

\section{KEYW/ORDS}

Competences profile, Occupation, Human Resources Director; Bebaviors, companies from Guayaquil. 


\section{Bntroducción}

$\mathrm{El}$ área de Recursos Humanos (RRHH) dentro de la empresa, y en general dentro de las organizaciones en Ecuador, ha evolucionado mucho en los últimos 12 años fruto de los cambios que ha experimentado el país no solo en el sector privado sino también en el público. Entre éstos pueden mencionarse a) los producidos en las leyes (la nueva Constitución de la República, el Mandato Constituyente No. 8, la nueva Ley Orgánica del Servicio Público, la creación de la remuneración unificada, la reforma a la ley para el pago mensual del fondo de reserva, el decreto para la inserción de personas con discapacidad en el ámbito laboral, la ley para la defensa de los derechos laborales de los empleados y trabajadores domésticos y el nuevo código de la producción que incorpora el concepto de salario digno), b) los cambios demográficos como el éxodo rural, la migración y el envejecimiento de la población activa, c) los económicos como la dolarización, el incremento en el precio del petróleo, la rápida evolución en las formas de organización, la internacionalización de las empresas d) los tecnológicos como el desarrollo e implantación masiva de las telecomunicaciones y la globalización de la información y e) la aparición de nuevas titulaciones universitarias orientadas a la gestión de los recursos humanos y la excesiva transformación de las universidades en centros de formación de técnicos y no de expertos e innovadores en sus disciplinas.

Asi mismo, el gestor de recursos humanos ha tenido que evolucionar para adaptarse a estas nuevas situaciones (PricewaterhouseCoopers, 2005; Elvira y Dávila, 2006; Aranguren, 2006). En Ecuador su formación profesional es algo relativamente reciente y suele estar a cargo de una institución de educación superior que le permite salir al mundo laboral con una base teórica que le faculta para asumir sus quehaceres profesionales de forma técnica y científica.
Estos cambios hacen que sea indispensable conocer cuál es el perfil de competencias que hoy en día se exige al director de recursos humanos ya que en opinión de Pereda, Berrocal y Sanz (2003) si no se conocen estas competencias es difícil definir los conocimientos que se deben adquirir y las habilidades y destrezas que se deben desarrollar.

Por otro lado, aunque se han realizado estudios sobre el perfil de competencias del profesional de RRHH (Brewster, Farndale y Ommeren, 2000; Pereda et al., 2003; Ulrich y Brockbank, 2006), ninguno de ellos lo aborda desde el contexto específico de Ecuador y más en concreto de Guayaquil.

Todas estas razones justifican un estudio que explore y describa el perfil de competencias que el director de recursos humanos de las empresas guayaquileñas debe tener, en el momento actual, para hacer frente a los desafíos de su profesión. Se utiliza, en cuanto a la forma de recoger los datos y a la forma de analizarlos, la metodología que Pereda et al.,(2003) aplicaron para determinar el perfil del profesional de recursos humanos y que también está descrita por Pereda, Berrocal y Alonso en su libro Psicología del trabajo (2008, -pp. 253-262).

\section{Marco Teórico}

\section{El director de recursos humanos}

Según Quijano (2006), para aclarar el rol del director de recursos humanos se consideran tres aspectos importantes que son: las áreas temáticas y contenidos de las tareas desarrolladas, la capacidad de tomar decisiones y de ejercer poder y la amplitud de la perspectiva o del enfoque adoptado.

Quijano (2006) considera tres tipos de profesionales: los de "personal", los de "relaciones laborales" y los de "recursos humanos". Los primeros se ocupan de la administración y control del personal. Su función 
es de tipo administrativo y realizan tareas operativas que no implican toma de decisiones. Los profesionales de "relaciones laborales" han sido tradicionalmente abogados y surgen ante la necesidad de la empresa de cumplir con los marcos legales en lo referente a las relaciones entre la empresa y el trabajador. En tercer lugar, los profesionales de "recursos humanos" son los que centran su actividad en el comportamiento individual y colectivo de los trabajadores. Sus funciones y actividades están enfocadas en diseñar y desarrollar procedimientos de selección, formación, evaluación, tetribución, o comunicación que desarrollen las potencialidades de los trabajadores en función de los objetivos de la organización (Quijano, 2006; Chiavenato, 2007). Estas actividades deben centrarse en conseguir trabajadores comprometidos con los objetivos de la empresa, motivados, competentes y eficaces en su desempeño y lo más satisfechos posibles en su vida laboral (Pérez Gorostegui, 2003).

En cuanto a los otros dos aspectos importantes que señala Quijano (2006), a saber, la capacidad de tomar decisiones y ejercer poder sobre cuestiones que afectan a la organización y la amplitud del enfoque adoptado centrada en problemas particulares o en una visión sistémica, se pueden distinguir dos roles distintos: el de técnico y el de director gerente.

El técnico es la persona que tiene conocimientos expertos sobre el área de su especialidad y puede resolver problemas concretos sin preguntarse sobre cómo influye la solución en el resto de la organización. Además no puede tomar decisiones sobre cuestiones estratégicas. Por su parte, el director - gerente tiene poder para tomar decisiones estratégicas, tiene también una visión global y sistémica de la organización, de su cultura y objetivos y del entorno del negocio en el que la organización se desenvuelve. Propo- ne ante el resto de la Alta Dirección de la empresa los objetivos de recursos humanos, en consonancia con los objetivos financieros, de mercado y de producción (Corral, 2009). Prevé la demanda de personal futura y propone y ejecuta acciones de contratación, formación, reubicación o desvinculación de personal (Quijano, Navarro, Yepes Baldó, Berger y Romeo Delgado, 2008). En definitiva, tiene poder para tomar decisiones sobre cuestiones que aportan resultados a la organización, desde un enfoque holístico y sistémico (Sastre y Aguilar, 2003).

\section{E1 concepto de competencia}

Las definiciones que se han dado del concepto de competencia han sido muchas (McClelland, 1961; Boyatzis, 1982; Spencer y Spencer, 1993; Bunk, 1994; Ducci, 1997; Leboyer, 1997; Mertens, 2000; Ruiz de Vargas, Romero y Jaraba, 2005; Blanco Prieto, 2007). La que se va a adoptar en este estudio es la propuesta por Pereda y Berrocal (2006, p. 21) quienes la definen como el "conjunto de comportamientos observables que están causalmente relacionados con un desempeño bueno o excelente en un trabajo concreto y en una organización concreta". Por tratarse de comportamientos observables, agrupados en categorías en función de sus similitudes, es posible medirlos. Por otra parte, el hecho de que cada organización y cada trabajo sean diferentes no quiere decir que sea imposible encontrar similitudes entre los comportamientos en perfiles de exigencias de trabajos similares. De ahí que se pueda hablar de la ocupación del director-gerente de recursos humanos, entendida como una función dentro de una organización, que engloba una serie de conductas que debe llevar a cabo y que le obliga a relacionarse con personas, herramientas, objetos y datos (Alcover de la Hera, Moriano, Osca y Topa, 2012; U.S. Departament of Labor/ Employment and Training Administration, 2013). 
Las competencias se pueden clasificar en competencias de organización, competencias ocupacionales, competencias de trabajo y competencias técnicas (Pereda et al., 2003). Este estudio se enfoca en las competencias ocupacionales relacionadas con el director de recursos humanos y son aquellas genéricas de la ocupación que estarían presentes en todos o en la mayoría de los trabajos o actividades que el director realiza.

\section{El perfil de competencias del director de recursos humanos}

La Federación Mundial de Asociaciones de Directores de Personal (WFPMA) (Brewster et al., 2000) elaboró una definición mundial de lo que es y lo que hace un profesional de recursos humanos en términos de "un conjunto global de competencias clave" (p. 23). Una de las principales conclusiones de ese estudio es que el conjunto de competencias clave no varían en los países participantes pero si hay variaciones en el peso que cada una de ellas tiene de acuerdo a las condiciones nacionales y organizacionales. Cabe notar que en este estudio no participó Ecuador. Las competencias identificadas por la WFPMA se agrupan en cuatro campos: estilo, implicación organizativa, liderazgo y técnicas. Dentro de éstos, se especifican competencias como credibilidad personal, conocimiento empresarial, creación de relaciones y conocimiento y práctica de la gestión de recursos humanos tradicional, por citar un ejemplo de competencias correspondientes a cada campo respectivamente.

Por otro lado Becker, Huselid y Ulrich (2002), definen las competencias como "las características individuales de conocimiento, capacidad, aptitud o personalidad" (p. 194), $y$ establecen un modelo de cinco campos de competencias (crediblidad personal, capacidad para gestionar cambios, capacidad para dirigir la política cultural, puesta en marcha de prácticas de recursos humanos y entendimiento del negocio) cada uno de los cuales contiene una serie de competencias específicas que son comportamientos observables. Posteriormente, Ulrich y Brockbank (2006), y Brockbank, Ulrich, Younger y Ultich (2012) propusieron un modelo de competencias basado en cinco categorías de competencias clave, que son las que agregan valor a la función de recursos humanos en la empresa. Las competencias clave de este modelo son la contribución estratégica, la credibilidad personal, la entrega de recursos humanos, el conocimiento del negocio y la tecnología de recursos humanos. Estos modelos se elaboraron a partir de muestras de directivos estadounidenses.

Por último, Pereda et al., (2003) también proponen un perfil de competencias clave, pero en este caso no para la ocupación del director de recursos humanos sino para el profesional técnico de los recursos humanos que no ocupa funciones de dirección. Para este tipo de profesionales, su perfil de exigencias incluye el trabajo en equipo, orientación al cliente, planificación/organización, comunicación, fexibilidad y colaboración. A cada una de estas competencias se asocian una serie de comportamientos específicos.

\section{Metodologia}

Objetivo

El objetivo de este estudio es conocer las competencias ocupacionales que forman el perfil de competencias del director de recursos humanos en empresas guayaquileñas.

\section{Muestra}

Se contactó a 400 personas que ejercían la función de dirección de RRHH de empresas domiciliadas en Guayaquil de las cuales 122 respondieron el cuestionatio (31\%). Por tratarse de un estudio exploratorio la selección se realizó de modo no probabilístico e incidental de acuerdo a la apertura que dieron los entrevistados para responder el cuestionario (Hernández, Fernández y Baptista, 
2006), tomando la base de datos de empresas domiciliadas en Guayaquil en el año 2011 de la Superintendencia de Compañías de Ecuador (2012).

De las 122 personas que respondieron el $43 \%$ son hombres y $57 \%$ mujeres. De ellos, el $14 \%$ están en empresas industriales, el $34 \%$ en empresas comerciales y el $52 \%$ en empresas de servicio. De acuerdo al tamaño de la empresa el $31 \%$ están en empresas pequeñas, $25 \%$ en medianas y $44 \%$ en grandes. Sus profesiones son variadas y comprenden desde abogado (9\%) hasta psicólogo clínico (4\%), siendo las más comunes ingeniero comercial $(26 \%)$ y licenciado en gestión de RRHH (26\%). También hay economistas $(11 \%)$, ingenieros técnicos $(10 \%)$ y licenciados en administración ( $9 \%$ ). Un $5 \%$ no tiene estudios superiores. En cuanto a la experiencia como directores de recursos humanos, $31 \%$ tienen poca experiencia (de 1 a 3 años), $46 \%$ tienen mediana experiencia (de 3 a 10 años) y $23 \%$ tienen mucha experiencia (más de 10 años).

\section{Procedimiento}

El cuestionatio consta de tres partes. La primera explica el objetivo de la investigación, pide la colaboración de los sujetos y recoge datos generales sobre el encuestado como sexo, profesión, años de experiencia en la función de dirección de RRHH, tamaño y tipo de empresa en la que trabajaba. La segunda comprende 25 competencias con sus respectivas definiciones, consideradas como relevantes para la ocupación de dirección de recursos humanos. Este listado se elaboró con base en la literatura revisada seleccionando todas las competencias de los diferentes perfiles propuestos por los autores y eliminando aquellas que se repetían. Ver tabla 1.

Se siguió la metodología propuesta por Pereda et al., (2003,) y Pereda et al., (2008) y se pidió a los encuestados que, de las competencias presentadas, seleccionaran las 10 que consideraban claves para que pudieran desempeñar sus funciones de forma eficaz, eficiente y segura. Después debían ordenar las diez competencias seleccionadas por orden de importancia.

La tercera parte del cuestionario incluye comportamientos asociados a las 25 competencias propuestas, cinco comportamientos para cada competencia. Del mismo modo que las competencias, estos comportamientos se seleccionaron con base en la literatura revisada. Un ejemplo de estos comportamientos puede verse en la tabla 2.

De los comportamientos presentados, los encuestados debían seleccionar un máximo de diez, los que considerasen más importantes para el director de RRHH y que correspondieran a las competencias que habían seleccionado en la segunda parte del cuestionario. Por último, debían ordenar los comportamientos elegidos del mismo modo que hicieron con las competencias.

Pereda et al., (2003) sugieren en sus conclusiones que la recogida de los datos se realice en las tres siguientes fases: a) presentar a los sujetos la tabla de las competencias, b) analizar las competencias seleccionadas $y$ elegir como relevantes las escogidas por al menos el $50 \%$ de la muestra y c) presentar a los sujetos únicamente los comportamientos asociados a las competencias relevantes. De esta manera se obligaría a los sujetos a elegir sólo entre los comportamientos de las competencias relevantes y no se perdería para el análisis a aquellos sujetos que eventualmente hubieran elegido comportamientos asociados a otras competencias no señaladas como relevantes. Sin embargo en este estudio se recogieron todos los datos en una sola fase. Se realizó un estudio piloto previo a quince personas para validar tanto el cuestionario 


\section{5:}

Elemplo de comporan enes asoclados que se incluyeronen a competencha

Aplicación de tecnología a RRHH

Utiliza el correo electrónico para la comunicación con el personal

Utilizaredes sociales para la comunicación con el personal

Utiliza sistemas informáticos para la gestión de las actividades de RRHH

como controles, nóminas

Utiliza la tecnologia para programas de formación

Utiliza la tecnología como base de datos del personal, inventario

de capacidades, etc

Fuente: Cálculos propios, elaborados a partir de datos de CEPAL, 2010

como la forma de recolección de los datos y el resultado reveló la dificultad de que los sujetos volvieran a contestar un segundo cuestionario en un momento posterior ${ }^{1}$ de manera que, de haber recogido los datos tal y como sugieren los autores mencionados, el índice de respuesta al segundo cuestionario (el que incluyera sólo los comportamientos) hubiera sido menor que recogiendo los datos en una sola fase, como en realidad se hizo.

\section{Análisis de los datos}

Siguiendo a Pereda et al., (2008, pp. 255260) para el análisis de los datos se obtuvieron dos puntuaciones: a) la Relevancia (R) que representa el porcentaje de encuestados que eligieron cada competencia como "clave" para la ocupación del director de recursos humanos. Esta puntuación indica el grado de acuerdo, entre los participantes, sobre la relevancia de cada competencia para la eficacia y la eficiencia de la labor de esta ocupación, y b) la Importancia (l) que se obtuvo a partir de las posiciones que los encuestados le asignaron a cada competencia como "clave" para el trabajo del director de recursos humanos. Este análisis se realizó tanto para las competencias como para los comportamientos asociados a cada competencia.

\section{Resultados y discusión}

Ptimero se mostrarán los resultados respecto de las competencias y después los referentes a los comportamientos asociados a ellas.
En cuanto a la relevancia de las competencias, los resultados generales del grupo evaluado se muestran en la tabla 3 .

Siguiendo a Pereda $e t$ al., (2003) se decidió que las competencias clave para el perfil del director de RRHH fueran aquellas elegidas por al menos el $50 \%$ de los encuestados, es decir, la mayoría de ellos. Estas son: liderazgo, desarrollo del personal, habilidades de comunicación, planificación/organización y negociación. A diferencia de los perfiles revisados en la literatura (Brewster et al., 2000; Becker et al., 2002; Pereda et al., 2003), la competencia negociación fue señalada como relevante para el director de recursos humanos por los sujetos de la muestra. Llegar a acuerdos satisfactorios para todos, utilizar estrategias y metodologías para diseñar y preparar ambientes de negociación $e$ identificar las diferentes posiciones en una negociación son comportamientos que un director de RRHH debe tener para ser exitoso en su desempeño.

Los resultados sobre la relevancia de las competencias varían según las características del grupo de acuerdo con las variables de género, actividad y tamaño de la empresa donde trabajan, profesión y años de experiencia dedicados a la ocupación de director de RRHH. Con respecto al género las diferencias en los resultados son mínimas. Solo notar que el grupo masculino, además de las cinco competencias señaladas, consideró también televante para su trabajo la aplicación de tecnologías a RRHH.

I La dificultad estriba en que la mayoría de los encuestados señalo que por sus múltiples ocupaciones como directores y la escasez de tiempo seria muy complicado para ellos volver a llenar otro cuestionario. (Sugerencia señalada por diez de los quince encuestados en la prueba piloto). 


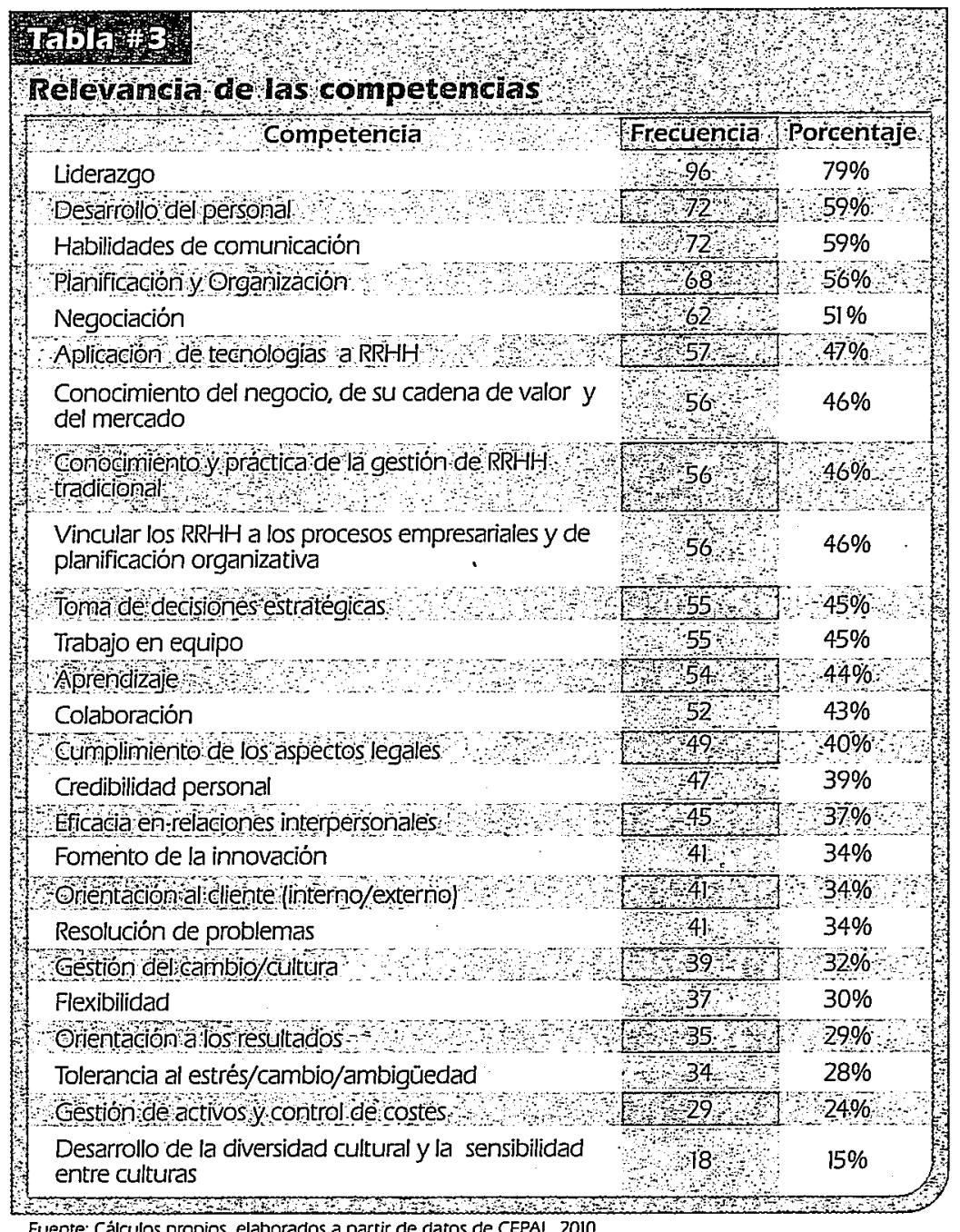

Fuente: Cálculos propios, elaborados a partir de datos de CEPAL, 2010.

Con respecto a la actividad de la empresa, de las cinco competencias elegidas como relevantes, solo el "liderazgo" es considerada por los directores de los tres tipos de empresa simultáneamente, habiendo también una diferencia importante entre las puntuaciones otorgadas ( $59 \%$ en las empresas industriales y $80 \%$ y $83 \%$ en las comerciales y de servicios respectivamente). Los encuestados de las empresas industriales seleccionaron la competencia "conocimiento del negocio, cadena de valor y del mercado" (59\%) y el "fomento de la innovación" y la "orientación al cliente" (53\%), y no seleccionaton el "desartollo del personal" y la "planificación/ organización". Los sujetos de empresas comerciales seleccionaron la "toma de decisiones estratégicas" (61\%) y el "aprendizaje" $(51 \%)$ y no seleccionaron la "habilidad de comunicación". Los de empresas de servicios seleccionaron "aplicación de tecnologías a RRHH" (56\%), "colaboración" (55\%), y "trabajo en equipo" (52\%) y no seleccionaron "negociación".

Con respecto al tamaño, las empresas grandes no consideraron relevantes ni la "planificación/ organización" ni la "negociación", lo cual llama la atención pues podría entenderse que a medida que aumenta el tamaño de la empresa también lo hace su complejidad y que, por tanto, esas competencias deberían ser consideradas claves. Por otro lado en las empresas pequeñas no se consideró relevante la "negociación".

La formación profesional de los encuestados es también una variable que influye bastante al seleccionar una competencia como relevante. Los resultados muestran una gran variedad de opiniones entre los directores de recursos humanos de acuerdo a su profesión. Por ejemplo, para el grupo de los abogados la "planificación y organización" es la competencia más relevante, sin embargo no seleccionaron ni las habilidades de comunicación ni la negociación. Para los economistas no es relevante la "planificación y organización" y el grupo de psicólogos no consideró relevante ni el "desarrollo del personal" ni la "planificación y organización" ni la "negociación". Para la mayoría de profesionales el "liderazgo" es la competencia 
más relevante de todas. Cabe señalar que de las 25 competencias propuestas, la única que no fue seleccionada como relevante por ningún grupo fue la "gestión del cambio y la cultura".

La experiencia de los encuestados en la función de dirección de recursos humanos es también un factor que discrimina al seleccionar una competencia como clave. Por ejemplo, el grupo con menos experiencia no seleccionó como clave la "negociación", mientras que el grupo de mayor experiencia no señaló como clave el "desarrollo del personal".

Para determinar la importancia de las competencias, así como su orden y ponderación final, resultante del producto de la relevancia por la importancia, se trabajó con las competencias señaladas como relevantes por el 50\% del grupo total de sujetos encuestados.

Los resultados de la tabla 4 muestran un pequeño cambio en el orden final de las competencias siendo la "planificación y organización" la competencia que está en tercer lugar mientras que la compe-

\section{Tabla \#4}

\section{Competencias del perfil del director de RRHH} ordenadas según su importancia

\begin{tabular}{|l|c|c|c|c|c|}
\hline & $\mathbf{R}$ & $\mathbf{I}$ & $\mathbf{R x I}$ & PONDERACIÓN & ORDEN \\
\hline Liderazgo & 79 & 678 & 53562 & $41,1 \%$ & 1 \\
\hline Desarrollo del personal & 59 & 387 & 22833 & $17,5 \%$ & 2 \\
\hline Habilidades de comunicación & 59 & 344 & 20296 & $15,6 \%$ & 3 \\
\hline $\begin{array}{l}\text { Planificación y organización } \\
\text { Negociación }\end{array}$ & 56 & 371 & 20776 & $15,9 \%$ & 4 \\
\hline TOTAL & 51 & 254 & 12954 & $9,9 \%$ & 5 \\
\hline
\end{tabular}

Fuente: Elaboración propia

\section{Tabla \# 5}

\section{Relevancia de los comportamientos}

\begin{tabular}{|c|c|}
\hline Liderazgo & $\mathrm{N}=96$ \\
\hline $\begin{array}{l}\text { Fija objetivos a su equipo, realiza su seguimiento y brinda retroalimentación } \\
\text { sobre su avance* }\end{array}$ & $67 \%$ \\
\hline Motiva e inspira confianza* & $50 \%$ \\
\hline Orienta la acción de su grupo en una dirección determinada & $46 \%$ \\
\hline $\begin{array}{l}\text { Desarrolla su energia y la transmite a otros en pos de un objetivo común } \\
\text { fijado por él mismo }\end{array}$ & $42 \%$ \\
\hline Inspira valores de acción y anticipa escenarios de desarrollo de la acción & $31 \%$ \\
\hline Desarrollo del personal & $\mathrm{N}=72$ \\
\hline $\begin{array}{l}\text { Se esfuerza constantemente por mejorar la formación y desarrollo tanto } \\
\text { personal como de los demás* }\end{array}$ & $66 \%$ \\
\hline $\begin{array}{l}\text { Da retroalimentación a los demás a fin de que sepan qué están haciendo } \\
\text { bien y si esto se adapta a lo esperado* }\end{array}$ & $58 \%$ \\
\hline Explica cómo y por qué las cosas se hacen de una determinada manera & $37 \%$ \\
\hline Facilita el aprendizaje de los demás & $31 \%$ \\
\hline $\begin{array}{l}\text { Se asegura por distintos medios de que se hayan comprendido bien sus } \\
\text { explicaciones e instrucciones }\end{array}$ & $28 \%$ \\
\hline Habilidades de comunicación & $\mathrm{N}=72$ \\
\hline Se comunica de forma rápida, clara y eficaz* & $55 \%$ \\
\hline $\begin{array}{l}\text { Se comunica bien tanto de manera escrita como interactuando con grupos } \\
\text { o individuos* }\end{array}$ & $52 \%$ \\
\hline Selecciona de forma adecuada los mensajes y los presenta claramente* & $50 \%$ \\
\hline Utiliza canales formales e informales de comunicación & $45 \%$ \\
\hline Envia mensajes claros respecto a lo que es importante para la organización y su éxito & $38 \%$ \\
\hline Planificación y Organización & $\mathrm{N}=68$ \\
\hline $\begin{array}{l}\text { Identifica y desarrolla los métodos apropiados para la consecución de los } \\
\text { objetivos del equipo o departamento y de la empresa* }\end{array}$ & $62 \%$ \\
\hline Establece objetivos ambiciosos pero realistas para el equipo o el departamento* & $60 \%$ \\
\hline $\begin{array}{l}\text { Provee los recursos (técnicos, humanos y económicos) para alcanzar los } \\
\text { objetivos en los plazos fijados* }\end{array}$ & $53 \%$ \\
\hline $\begin{array}{l}\text { Anticipa los puntos críticos de una situación con un gran número de } \\
\text { variables, estableciendo puntos de control, verificando datos y buscando } \\
\text { información para asegurar la calidad del proceso }\end{array}$ & $38 \%$ \\
\hline Administra simultáneamente diversos proyectos complejos & $34 \%$ \\
\hline Negociación & $\mathrm{N}=62$ \\
\hline Lega a acuerdos satisfactorios para todos* & $60 \%$ \\
\hline $\begin{array}{l}\text { Utiliza estrategias y metodologías para diseñar y preparar ambientes de } \\
\text { negociación* }\end{array}$ & $50 \%$ \\
\hline Identifica las diferentes posiciones en una negociación* & $50 \%$ \\
\hline Se centra en el problema y no en la persona & $40 \%$ \\
\hline Logra la colaboración y compromisos duraderos que fortalecen las rela & $22 \%$ \\
\hline
\end{tabular}


tencia "habilidades de comunicación quedó en el cuarto lugar.

Con respecto a los comportamientos se empleó el mismo método de análisis que para las competencias y sólo se tuvieron en cuenta aquellos comportamientos asociados a las competencias señaladas como relevantes y seleccionados por al menos el $50 \%$ de los evaluadores. La tabla 5 muestra la rele- vancia de los comportamientos de cada competencia. Hay que señalar que el " $n$ " de cada competencia varía según el número de sujetos que las habían seleccionado como relevante.

Por último, en la tabla 6 se muestra el perfil de competencias de la ocupación del director de recursos humanos con los comportamientos asociados a cada competencia

$\mathrm{y}$ sus ponderaciones respectivas.

\section{$16 \mathrm{blat}$}

Perrilde comperencias de la ocupachondel Director de RRH

GOMPETENCIA Ponderacion COMPORTAMIENTOS Ponderación

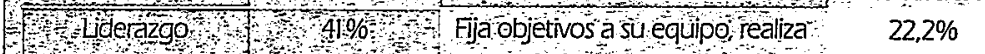

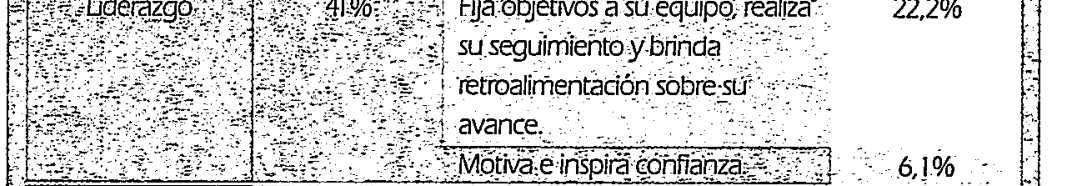

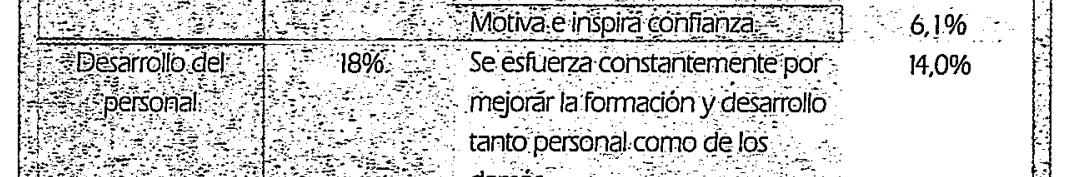

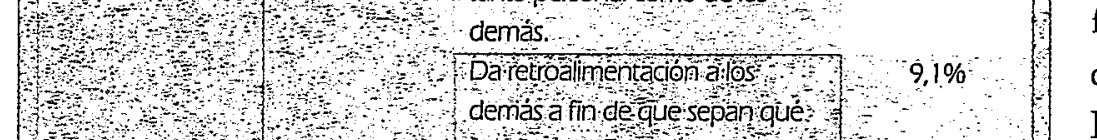

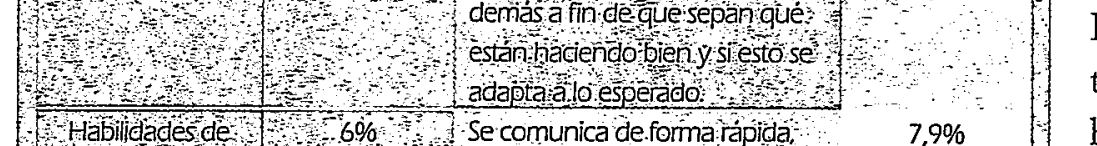

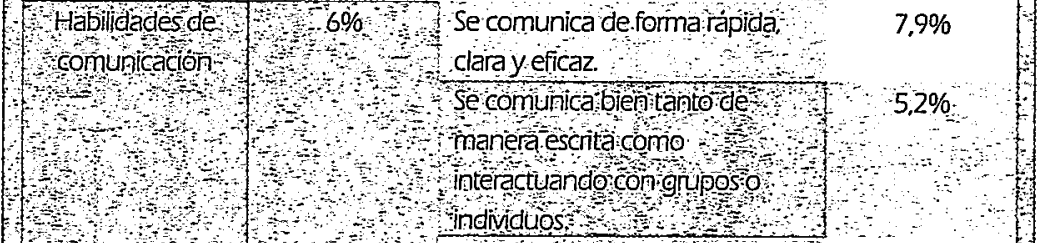

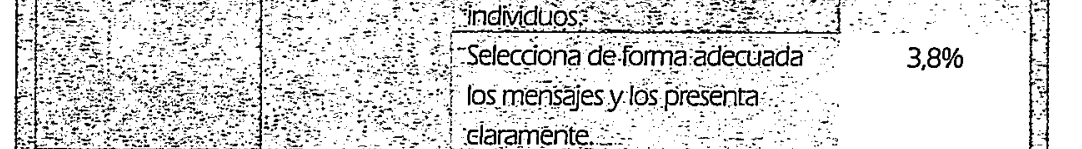

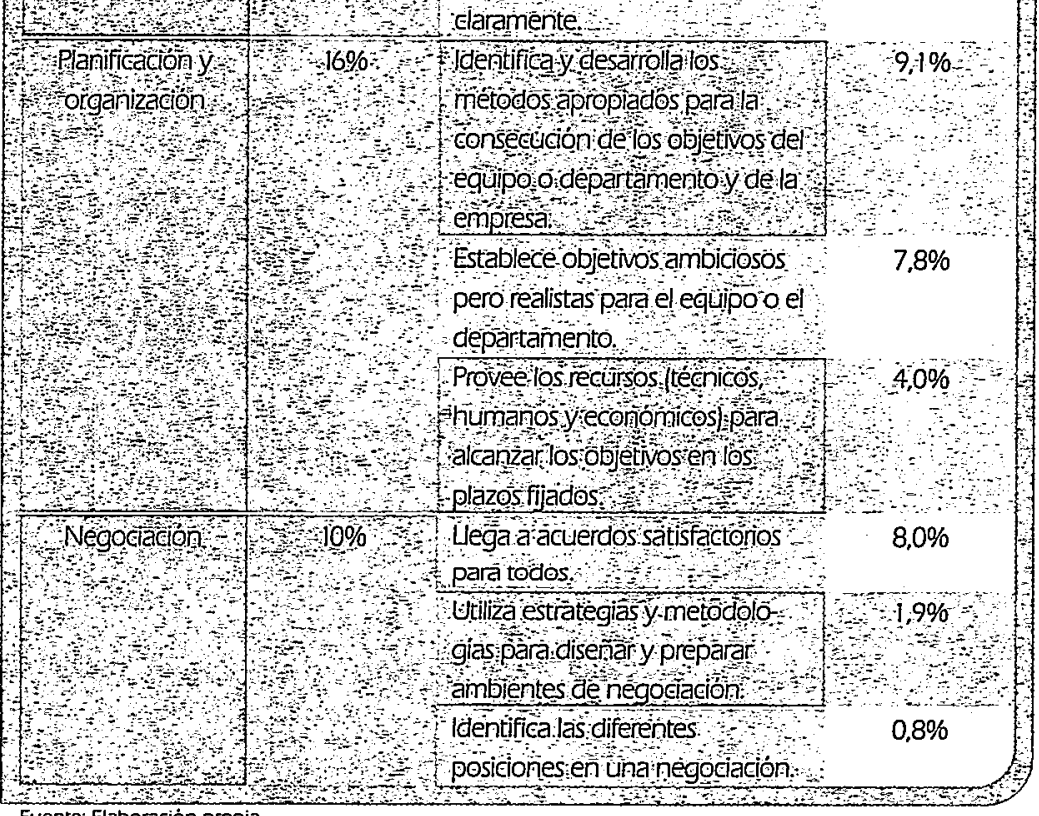

Fuente: Elaboración propia.

\section{Conclusiones}

Este estudio pretende incursionar en la línea de investigación señalada por Pereda et al., (2003), aportando con una descripción sobre el perfil de competencias del director de RRHH en Ecuador y concretamente en Guayaquil, donde hasta ahora no se habían realizado estudios de este tipo, y cuyos resultados contribuirán a desarrollar la actividad del director de recursos humanos como función importante y estratégica dentro de las organizaciones guayaquileñas y ecuatorianas.

En cuanto a la metodología empleada se comprueba, siguiendo a Pereda et al., (2003) que es adecuada para elaborar el perfil de competencias ocupacional del director de recursos humanos. También se validó que la recogida de datos en una sola fase es 
pertinente a pesar de la sugerencia de los autores citados de hacerlo en varias fases para no perder sujetos de la muestra. Sin embargo hacerlo en varias fases hubiera supuesto una pérdida mayor.

Se establece el perfil de competencias con sus comportamientos asociados ordenados según su importancia y peso para el director de recursos humanos de empresas pequeñas medianas y grandes y de los tres sectores de actividad de Guayaquil. Este perfil puede tener sus variantes de acuerdo con el tipo de empresa y con las características propias de quien ocupa la función de dirección (género, experiencia profesional o estudios). Esto confirma que aunque las competencias son específicas para cada puesto y para cada organización es posible establecer un perfil de competencias ocupacionales generales basados en la similitud de los comportamientos (Pereda y Berrocal, 2006; Alcover de la Hera et al., 2012).

Pero también se puede decir que no existe el perfil ideal de competencias, aquel que llevaría a un comportamiento exitoso en cualquier organización independientemente del lugar y del tiempo (Becker et al., 2002; Ulrich y Brockbank, 2006). La rapidez con la que se producen los cambios en las organizaciones y las diferencias espacio-temporales obligan a la flexibilidad y adaptación. Por tanto, cualquier perfil de competencias que se proponga deberá tener en cuenta esta variabilidad.

Llama la atención que, aunque la gestión del cambio es uno de los principales desafíos que se le presenta a la función de dirección de recursos humanos con base en los cambios experimentados en el país y de acuerdo a lo señalado por algunos autores (Blasco, 2004; PricewaterhouseCoopers, 2005) sin embargo, no haya sido elegida como competencia relevante. Es más, de las 25 competencias presentadas fue la única no elegida como relevante por ninguno de los subgrupos en que se clasificó a la muestra. Podría pensarse que se trata de una competencia que ya está contenida en otras y que podría eliminarse del cuestionario para futuras investigaciones, o que quizá en el momento actual del país no se considera relevante para el director de RRHH.

Aunque el concepto de ocupación utilizado en el estudio hace referencia a comportamientos y no a títulos profesionales, el análisis de las competencias sugeridas como relevantes para el director de recursos humanos, según la profesión de los encuestados, manifiesta una gran variabilidad. Podría decirse que, si la formación científico-técnica de los estudios profesionales hace que se priorícen unos comportamientos sobre otros, entonces la formación del director de recursos humanos debe resaltar los aspectos referentes al comportamiento humano, tanto individual como grupal, en un ambiente laboral, para garantizar un comportamiento de acuerdo a lo que su ocupación exige.

Este estudio limita su alcance a la ciudad de Guayaquil. Extender el estudio a nivel nacional podrá dar una visión más completa del perfil de competencias de director de RRHH. También se sugiere, como futura investigación, extender el alcance a otros profesionales del área para poder comparar resultados con estudios como los de la WFPMA (Brewster, et al., 2000).

\section{Bibliografía}

Alcover de la Hera, C., Moriano, J., Osca, A. y Topa, G. (2012). Psicología del Trabajo. Madrid: UNED Editorial.

Aranguren, A. W. (2006). Gestión de recursos humanos y globalización. Visión gerencial, 5 (2), 113-121.

Becker, B., Huselid, M. y Ulrich, D. (2002). $E$ l cuadro de mando de RRHH, vinculando a 
las personas, la estrategia y el rendimiento de la empresa. Barcelona: Gestión 2000.

Blanco Prieto, A. (2007). Trabajadores competentes, introduccion y reflexiones sobre la gestion de recursos bumanos por competencias. Madrid: ESIC Editorial.

Blasco, R. (2004). Reclutamiento y selección, viejo y nuevo rol del psicólogo. Revista de Psicologia de la Universidad de Barcelona, 91-122.

Boyatzis, R. (1982). The competent manager. New York: Wiley \& Sons.

Brewster, C.,Farndale, E. y Ommeren, J. (2000). Competencias y estándares profesionales para la dirección de Personal/Recursos Humanos. París: AEDIPE, FUNDIPE, AMEDIRH.

Brockbank, W., Ultich, D., Younger, J. y Ulrich, M. (2012). Recent study shows impact of HR competencies on business performance. Employment Relations Today, 39, 1-7. doi: 10.1002/ert.21348.

Bunk, G. (1994). La transmisión de las competencias en la formación y perfeccionamiento profesionales en la RFA. Revista Europea de la Formación Profesional, I, 8-14.

Chiavenato, I. (2007). Administración de recursos humanos. México: McGraw Hill.

Compañias, S. d. (25 de julio de 2012). Superintendencia de Compañias. Recuperado de http://www.infoempresas.supercias. gov.ec/ibmcognos/cgi-bin/cognosisapi.dll?b_action $=x t s . r u n \& m=$ portal $/$ cc.xts\&m_tab $=$ i4F360B08331945A3883 AAF5781C1E281

Corral, J. (2009). El director de Recursos Humanos como CEO. Capital Humano, N. 231, 58-60.
Ducci, M. (1997). El enfoque de competencia laboral en la perspectiva internacional. Montevideo: Cinterfor/OIT.

Elvira, M. y Dávila, A. (2006). Retos emergentes para la investigación de la administración de Recursos Humanos en America Latina. Revista de Empresa n 15, 26-36.

Hernández, Fernández y Baptista. (2006). Metodologia de la investigación. México: McGraw Hill.

Leboyer, L. (1997). Gestión de las competencias. Barcelona: Gestión 2000.

McClelland, D. (1973). Testing for Competencies rather than Inteligence. American Psychologist, 28, 1-14.

Mertens, L. (2000). La gestión por competencia laboral en la empresa y en la formación profesional. Recuperado de: www.cinterfor.org. uy. $5 / 1 / 2012$

Pereda, S. , Berrocal, F. y Sanz, P. (2003). Los perfiles de exigencias en la ocupación del profesional de Recursos Humanos. Psicologia desde el Caribe, Universidad del Norte, $\mathrm{N}$. 12, 13-38.

Pereda, S. y Berrocal, F. (2006). Técnicas de gestion de recursos bumanos por competencias. Madrid: Editorial Centro de Estudios Ramón Arecés.

Pereda, S., Berrocal, F. y Alonso, R. (2008). Psicologia del trabajo. Madtid: Editorial Síntesis.

Pérez Gorostegui, E. (2003). Dirección de Recursos Humanos en la Empresa. Madrid: UNED.

PricewaterhouseCoopers. (2005). Encuesta sobre desafios globales de RRHH: ayer, boy $y$ 
mañana. Recuperado de: PricewaterhouseCoopers: www.pwc.com. 30/10/2011

Quijano, S. (2006). Dirección de Recursos Humanos y Consultoria en las organizaciones. Barcelona: Icaria Editorial S.A.

Quijano, S., Navarro, J., Yepes Baldó, M., Berger, R. y Romeo Delgado, M. (2008). La auditoría del sistema humano (ASH) para el análisis del comportamiento humano en las organizaciones. Papeles del Psicólogo, vol. 28, (1), 92-106.

Ruiz de Vargas, M., Romero, L y Jaraba, B. (2005). Competencias laborales y la formación universitaria. Psicología desde el Caribe, 16, 64-91.

Sastre, M. y Aguilar, E. (2003). Dirección de recursos bumanos, un enfoque estratégico. Madrid: McGraw Hill.

Spencer, L.M. y Spencer, S. (1993). Competence at work: Models for superior performance. New York: Wiley \& Sons.

U.S. Departament of Labor/ Employment and Training Administration. (2013). O*NET Resource Center (Occupational Information Network). Recuperado de http:// www.onetcenter.otg 23/8/2013

Ulrich, D. y Brockbank, W. (2006). La propuesta de valor de Recursos Humanos. Bilbao: Deusto.

\section{Jose Antonfo a hat Ahoy}

Mástér en Direción de Re eursos llumanos Profesor dela facultad de Ecología lumana y Desar rollo de Ta Universiedad Casa Gandé Guayaquil, Egtador.

Ciudadela Míafleres Av las Palmas $304 \mathrm{y}$ cälle 4 ?

\section{E-mail jgareaecasägradededue}

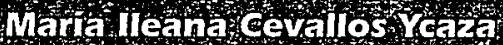

Ingeniera en gestion de Recursos Humanos. Investigadora de la Facuiltad de Ecología Humana y Desarrollo de la Universidad easa Grande, Glayaquil Eclador

Giudadela Miratiores, Av las Palmas 304 y callé 4 a

E-mail naileanacevallosyegmail com 
